\title{
Carbonaceous dust grains in luminous infrared galaxies
}

\section{Spitzer/IRS reveals a-C:H as an abundant and ubiquitous ISM component ${ }^{\star}$}

\author{
E. Dartois ${ }^{1}$ and G. M. Muñoz-Caro ${ }^{2}$ \\ ${ }^{1}$ Institut d'Astrophysique Spatiale (IAS), Université Paris-Sud 11, CNRS (UMR 8617), Bâtiment 121, 91405 Orsay, France \\ e-mail: emmanuel.dartois@ias.u-psud.fr \\ 2 Centro de Astrobiología, INTA-CSIC, Carretera de Ajalvir, km. 4, Torrejón de Ardoz, 28850 Madrid, Spain
}

Received 5 May 2007 / Accepted 18 July 2007

ABSTRACT

\begin{abstract}
Aims. The available ground- and space-based spectroscopic capabilities of observatories now allow us to extend Galactic interstellar medium composition studies to extragalactic cases. Absorptions in the mid-infrared shows evidence for silicate and carbonaceous grains in other galaxies.

Methods. A set of extragalactic spectra of luminous infrared galaxies (LIRGs) has been extracted from the Spitzer database and compared to the spectra of laboratory-produced interstellar carbon dust analogues.

Results. These highly obscured lines-of-sight display the characteristic absorptions at $\sim 6.85$ and $7.25 \mu \mathrm{m}$ of the $\mathrm{CH}_{3} / \mathrm{CH}_{2}$ deformation modes of hydrogenated amorphous carbon $(\mathrm{a}-\mathrm{C}: \mathrm{H})$ grains. They are compared to laboratory-produced a-C:H and imply carbon atom column densities in the solid phase exceeding $\sim 10^{18} \mathrm{~cm}^{-2}$.

Conclusions. These observations further demonstrate the ubiquitousness of a-C:H in the diffuse interstellar medium (DISM) of galaxies, for a long time almost only observed in the Milky-Way ISM lines-of-sights. Whereas PAH emission lines trace the reprocessing of energetic young stellar radiation, the observed a-C:H features underline the existence of large masses of amorphous carbon dust in (extra-)galactic dust budgets. The difficulty in observing such an interstellar component in the mid-infrared is linked to its low absorption contrast $\left(A_{\mathrm{V}} / \tau(6.85) \approx 625 \pm 40\right)$ for the strongest band, which therefore requires high column densities to detect a-C:H grains. Such carbon grains might be present but spectroscopically hidden in many other galactic environments.
\end{abstract}

Key words. ISM: dust, extinction - galaxies: ISM - methods: laboratory

\section{Introduction}

Measuring the spectral energy distribution of galaxies is an important observational task to understand not only how they are distributed, how they evolve and predict/subtract foreground contributions for high $z$ cosmological parameters determination, but also their chemical evolution. Space borne instruments with a good overall sensitivity and spectral resolution allow us to perform spectroscopic observations which resolve the different chemical components in external galaxies.

Prior to the Infrared Space Observatory satellite (ISO), midinfrared ground-based studies were often dedicated to the strong silicate Si-O stretching mode absorption. The ISO spectrometers began to look at the deeply-obscured mid-infrared spectra of galaxies (e.g. Lutz et al. 1998; Genzel et al. 1998; Rigopoulou et al. 1999; Tran et al. 2001; Spoon et al. 2001, 2002; Dartois et al. 2004a), starting with a few of the most luminous objects of their class and revealing the occurrence of ice and carbonaceous dust features in these extragalactic lines-of-sight. The Spitzer satellite (Werner et al. 2004), with its much higher sensitivity, is providing the basis for a statistical investigation of a large number of external galaxies. The atlas of mid-infrared galaxy spectra for intrinsically luminous but weak obscured AGNs is

* This research has made use of the SIMBAD database, operated at CDS, Strasbourg, France, and of the NASA/IPAC Extragalactic Database (NED) which is operated by the Jet Propulsion Laboratory, California Institute of Technology, under contract with the National Aeronautics and Space Administration. an opportunity to probe the chemical composition of dust in a large sample of galaxies (e.g. Spoon et al. 2007; Imanishi et al. 2007; Armus et al. 2007; Sajina et al. 2007).

In this article we explore the possibility of observing extragalactic hydrocarbon features at $6.85\left(\mathrm{CH}_{2} / \mathrm{CH}_{3}\right.$ deformation) and $7.25 \mu \mathrm{m}\left(\mathrm{CH}_{3}\right.$ sym. deformation), the counterpart of the $3.4 \mu \mathrm{m}$ (CH stretching modes) absorption observed in our Galaxy (e.g. Sandford et al. 1991; Pendleton et al. 1994) and thus to trace a significant fraction of carbonaceous dust in the ISM of galaxies. We first describe (Sect. 2) the observations retrieved from the Spitzer archive. In Sect. 3, we derive the hydrocarbon column densities, describe the status of other absorptions related to the carbonaceous dust backbone, investigate the correlation with inorganic dust (silicates) and discuss the galaxy sample where a-C:H is found. The conclusions are given in Sect. 4.

\section{Observations}

The observations released and analysed in this article were retrieved from the Spitzer database for various Spitzer/IRS programs, dedicated to the spectroscopic study of luminous galaxies classified as HII, Seyferts, interacting pairs, ULIRGs and LINERs galaxies. Table 2 lists the sources extracted from the Spitzer programs ${ }^{1}: 69$ (A Mid-IR Hubble Atlas of Galaxies,

\footnotetext{
${ }^{1}$ Readers are invited to consult the Spitzer website http:// ssc.spitzer.caltech.edu/ to obtain detailed information on these numerous programs.
} 
PI: Giovanni Fazio), 86 (Imaging and Spectroscopy of X-ray Selected Seyfert Galaxies, PI: Michael Werner), 96 (The mid-IR SED of nearby AGN, PI: James R. Houck), 105 (Spectroscopic Study of Distant ULIRGs II, PI: James Houck), 159 (SINGS: The Spitzer Infrared Nearby Galaxies Survey - Physics of the Star-Forming ISM and Galaxy Evolution, PI: Robert Kennicutt), 1096 (Dust in ULIRG environments, PI: Henrick W Spoon), 1430 (The Evolution of Activity in Massive Gas-Rich Mergers, PI: Sylvain Veilleux), 1421,1430,1438 (SIRTF IRS Calibration Program, PI: Lee Armus), 2306 (Buried AGNs in ultraluminous infrared galaxies - A search for a strong dust temperature gradient, PI: Masatoshi Imanishi), 3187 (The Evolution of Activity in Massive Gas-Rich Mergers, PI: Sylvain Veilleux), 3269 (Infrared SEDs of Seyfert Galaxies: Starbursts and the Nature of the Obscuring Medium, PI: Jack F Gallimore), 20375 (Of Starbursts and Monsters: An IRS High-resolution Spectroscopic Study of Ultraluminous Infrared Galaxies, PI: Lee Armus), 30323 (A Spitzer Spectroscopic Survey of a Complete Sample of Luminous Infrared Galaxies in the Local Universe, PI: Lee Armus). All these galaxies have in common an absorption spectrum with a rising slope in the mid-infrared. In addition, some ISO/SWS spectra and VLT/ISAAC were also included in the analysis, but are not shown here.

The observations mainly consist of IRS (Houck et al. 2004) Short-Low (SL) and Long-Low (LL) spectra, but a few also include higher resolution Short-High (SH) and Long-High (LH) IRS data. Using the SMART v6.2.4 package (Higdon et al. 2004) some spectra were interactively extracted using a background subtraction. For others we checked that the PBCD backgroundsubtracted spectra provided by the pipeline were of sufficient quality for the analysis presented in this article. After this, the orders were rescaled and stitched together with typical gain correction factors of the order of $5-10 \%$.

The Spitzer spectra in the 5.5-14 $\mu \mathrm{m}$ wavelength range, corrected for their redshift, as given in the Nasa Extragalactic or Simbad databases ${ }^{2}$ and given in Table 2, are displayed in Fig. 1 and ordered by decreasing $6.85 \mu \mathrm{m}$ optical depth from top left to bottom right. The main emission and absorption features occurring in this range are given in Table 1.

A gray stripe is drawn to delineate the region where the strongest $\mathrm{CH}_{3} / \mathrm{CH}_{2}$ deformation absorptions, around $6.85 \mu \mathrm{m}$, are expected. A close-up of the 5.5-7.5 $\mu \mathrm{m}$ window is presented in Fig. 2 in optical depth after division by a first order local continuum estimated on either side of the 6.85 and $7.25 \mu \mathrm{m}$ absorption.

\subsection{List of sources}

The sources presented in Table 2 are ordered into two subclasses following the detection of the infrared characteristic features of the a-C:H dust component. Class A comprises sources where both the $\mathrm{L}$ band $(3.4 \mu \mathrm{m})$ and $6.85 / 7.25 \mu \mathrm{m}$ absorption features are detected. The second class (B) are sources with only Spitzer $6.85 / 7.25 \mu \mathrm{m}$ absorption features detected. Note that the sources in class A are often less obscured. This can be explained by the fact that, due to the high extinction, some class B sources are rather opaque at $3.4 \mu \mathrm{m}$, since $\tau(6.85) / \tau(3.42) \approx 0.4$ (see Sect. 3.1). A few additional promising sources with low $\mathrm{S} / \mathrm{N}$ detection were not included, because we are awaiting higher signal-to-noise observations.

\footnotetext{
${ }^{2}$ http://nedwww.ipac.caltech.edu/ and http://simbad.u-strasbg.fr/simbad/
}

\section{Results and discussion}

\subsection{Column densities and constraints}

As discussed by Dartois et al. (2007) the $6.85 \mu \mathrm{m}$ absorption feature cannot be ascribed to a single vibration as it is a blend of methyl and methylene modes. As a consequence, the integrated absorption cross-section for this band corresponds to the overlap of several contributions. However, a mean integrated absorption cross-section can be evaluated from laboratory-produced analogues spectra, using as a reference the $\mathrm{CH}_{3}$ symmetric deformation mode at $\sim 7.25 \mu \mathrm{m}$ with an integrated absorption cross-section $A(7.25 \mu \mathrm{m}) \approx 0.75-1.5 \times 10^{-18} \mathrm{~cm} / \mathrm{CH}_{3}$ functional group (e.g. Wexler 1967; Spoon et al. 2004; Dartois et al. 2007). The integrated absorbance ratio between the 6.85 and $7.25 \mu \mathrm{m}$ absorption features in the laboratory spectrum presented at the top of Fig. 1 is evaluated to be $4 \pm 0.5$. The spectrum has a $\mathrm{CH}_{2} / \mathrm{CH}_{3}$ ratio of about 2 (estimated from the $3.4 \mu \mathrm{m}$ asymmetric stretching bands) and a contribution from both methyl and methylene groups to the $6.85 \mu \mathrm{m}$ absorption, which translates into a mean integrated absorption cross-section of $A(6.85 \mu \mathrm{m}) \approx 1.5 \pm 0.5 \times 10^{-18} \mathrm{~cm} / \mathrm{CH}_{n=2,3}$ functional group.

The $6.85 \mu \mathrm{m}$ absorption full width at half maximum (FWHM) lies between 35 and $40 \mathrm{~cm}^{-1}$ (corresponding to $\approx 0.18 \pm 0.02 \mu \mathrm{m})$. The optical depth can be related to the $\mathrm{CH}_{n=2,3}$ functional group column density along the line-of-sight by $N\left(\mathrm{CH}_{n=2,3}\right) \approx 2.5 \pm 0.7 \times 10^{19} \tau(6.85) \mathrm{cm}^{-2}$.

If one compares this value to the visual extinction relation found for the local diffuse ISM of $A_{\mathrm{V}} / \tau(3.42)=250 \pm$ 40 (Pendleton et al. 1994; Sandford et al. 1995), and using the laboratory measured intensity ratio $\tau(6.85) / \tau(3.42) \approx 0.4$, then $A_{\mathrm{V}} / \tau(6.85) \approx 625 \pm 40$. Such a low contrast explains the difficulty in observing hydrogenated amorphous carbon interstellar grains via these modes. If one assumes a local interstellar $A_{\mathrm{V}} / N_{\mathrm{H}}=4.48 \times 10^{-22} \mathrm{~cm}^{2}$ (Ryter 1996), one obtains the total hydrogen column density relation $N_{\mathrm{H}} \approx 1.4 \times$ $10^{24} \tau(6.85) \mathrm{cm}^{-2}$.

\subsection{Other absorptions}

In the infrared spectra of the sources considered here there may also be contributions from interstellar ice mantles (predominantly the bending mode of water ice) which can overlap with the carbonaceous matter absorption (e.g. IRAS F00183-7111, Spoon et al. 2004). Whereas, along other lines-of-sight devoid of strong ice absorptions (e.g. IRAS 08572+3915, Dartois et al. 2007) an additional absorption between 5.8 and $6.7 \mu \mathrm{m}$ can be attributed to the carbonaceous structural backbone, with $\mathrm{sp}^{2}$ bonding character and linking the observed methyl $\left(\mathrm{CH}_{3}\right)$ and methylene $\left(\mathrm{CH}_{2}\right)$ to form a solid network. In this case, additional carbon to that observed through the $\mathrm{CH}_{n=2,3} 6.85 \mu \mathrm{m}$ and $7.25 \mu \mathrm{m}$ features is necessary. The observed carbon $\mathrm{C}-\mathrm{H}$ bending modes therefore represent only a lower limit to the total carbon content of the a-C:H. The possible structures participating in the 5.8 to $6.7 \mu \mathrm{m}$ absorption include carbonyl $(\mathrm{C}=\mathrm{O})$, linear $\mathrm{C}=\mathrm{C}$ (olefinic) and cyclic $\mathrm{C}=\mathrm{C}$ (aromatic). Unless an independent observation of the $3 \mu \mathrm{m}$ ice absorption can confirm and establish an estimate of the amount of ice present in the $6 \mu \mathrm{m}$ region (as in NGC 4418), it is hard to disentangle the two contributions, except when one of the two components dominates, because the ice band peak position is at $\sim 6.0 \mu \mathrm{m}$ while the carbonaceous network absorption peaks at longer wavelengths. 

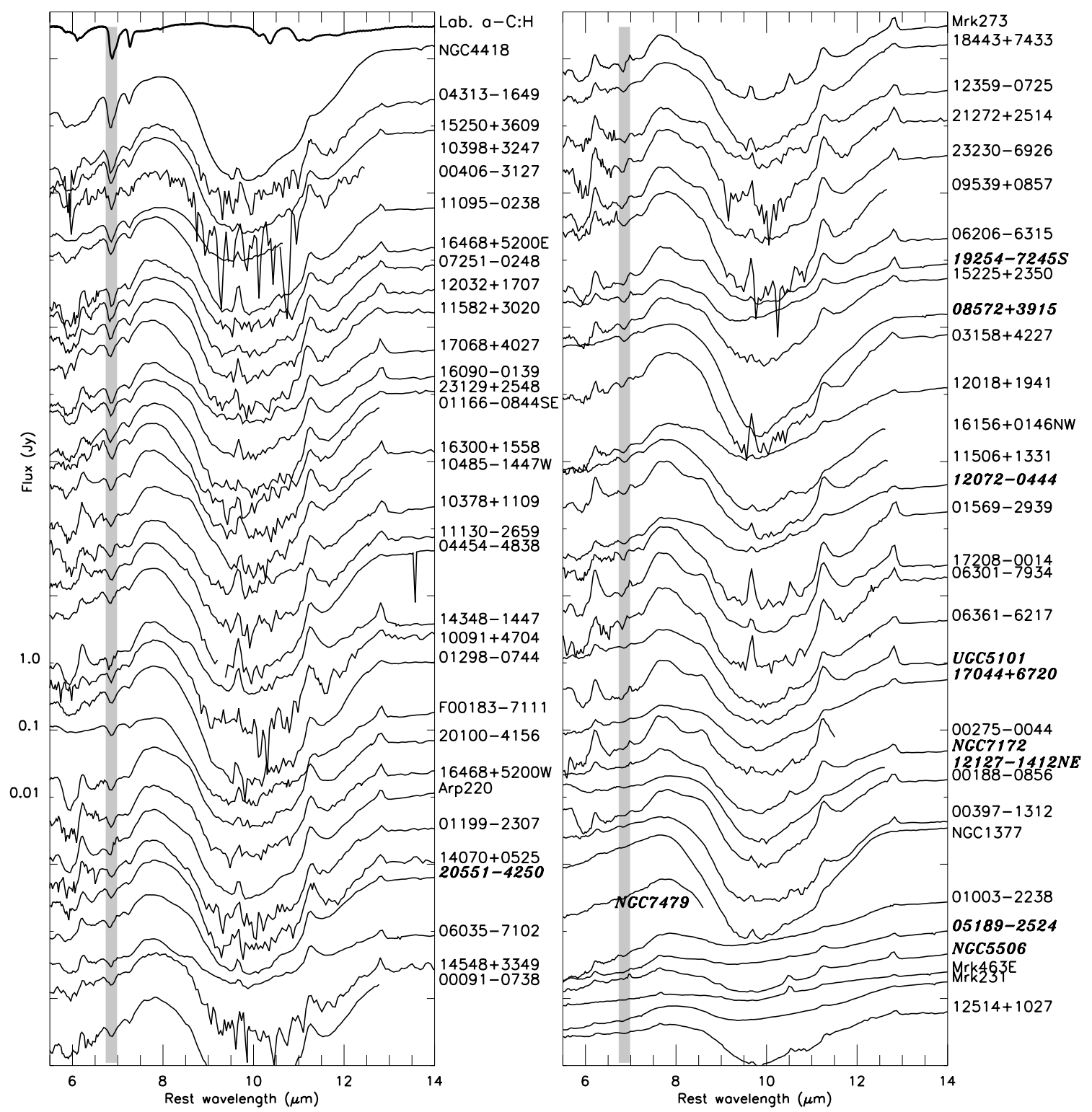

Fig. 1. Spitzer galaxy spectra in the 5.5-14 $\mu$ m range showing the a-C:H carbonaceous 6.85 and $7.25 \mu \mathrm{m}$ absorptions, ordered by decreasing $6.85 \mu \mathrm{m}$ optical depth. The top left spectrum is a laboratory-produced analogue displayed for comparison (see Dartois et al. 2005, for details). All spectra have been redshift-corrected using the values given in Table 2. A gray stripe delineates the expected range for the strongest carbonaceous absorption at $6.85 \mu \mathrm{m}$. The source names are given on the right of each spectrum, those in bold italic correspond to lines-of-sight where the $3.4 \mu \mathrm{m}$ hydrocarbon absorption is also observed.

\section{3. a-C:H versus silicates correlation}

A long standing and open question relating to diffuse Galactic matter is the structural association between the inorganic (silicates) and organic carbonaceous dust. In particular, interstellar dust grains are often thought to consist of a silicate-core covered by an organic-mantle, making a direct physical link between the carbonaceous matter and the silicates. One example is the Greenberg model (e.g. Greenberg et al. 1995) in which organic mantles on silicate grains were formed by the processing of ice mantles in dense clouds (first generation organics), followed by further processing in the diffuse medium (second generation organics). Another type of model proposed two separate populations of organics and silicate grains (e.g. Draine 2003, and references therein). In the prototypical Seyfert 2 NGC 1068, recent polarimetric observations seem to put into question the strongly correlated organic/inorganic model (Mason et al. 2007;
Table 1. Dominant features in the observations.

\begin{tabular}{lcl}
\hline \hline Rest wavelength $(\mu \mathrm{m})$ & Component & \\
\hline 4.27 & $\mathrm{CO}_{2}$ & abs. \\
6.00 & $\mathrm{H}_{2} \mathrm{O}$ ice & abs. \\
6.20 & PAH & emi. \\
6.85 & $\mathrm{a}-\mathrm{C}: \mathrm{H}$ & abs. \\
7.25 & $\mathrm{a}-\mathrm{C}: \mathrm{H}$ & abs. \\
9.66 & $\mathrm{H}_{2} \mathrm{~S}(3)$ & emi. \\
9.7 & Silicates & abs. \\
11.3 & PAH & emi. \\
12.7 & PAH & emi. \\
\hline
\end{tabular}

see also Chiar et al. 2006, for Milky Way studies), but this clearly requires further observational constraints.

Extragalactic lines-of-sight offer the opportunity to test if a tight correlation exists between carbonaceous a-C:H dust and 

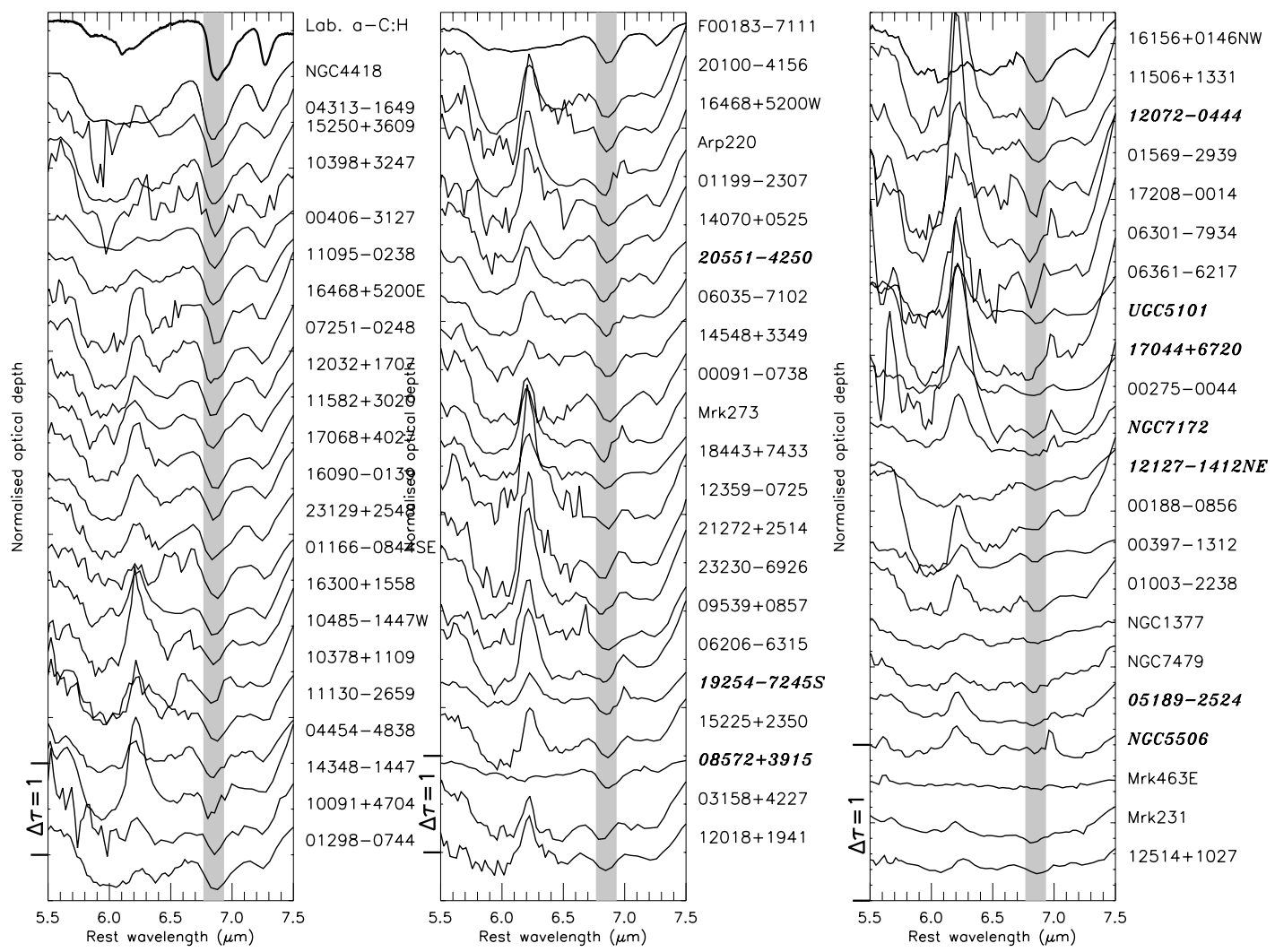

Fig. 2. Optical depth spectra in the 5.5 to $7.5 \mu \mathrm{m}$ range obtained by dividing the spectra presented in Fig. 1 by a local continuum (estimated on each side of the range displayed). A gray stripe delineates the spectral range for the strongest carbonaceous absorption at $6.85 \mu \mathrm{m}$. For clarity, the labels correspond to the observed galaxy spectra of Fig. 1.

silicate dust column densities, which would then imply at least co-spatial grain populations. The comparison of the $10 \mu \mathrm{m}$ silicate absorption along the same lines-of-sight is made easier with the $6.85 / 7.25 \mu \mathrm{m}$ features, as the bands are closer to the silicate feature than the $3.4 \mu \mathrm{m}$ absorption feature. The underlying continuum should therefore display similar spatial extent, thus considerably lowering radiative transfer effects when looking for a correlation. In addition, the vast majority of the sources presented here have a $10 \mu \mathrm{m}$ silicate optical depth greater than unity, ensuring us that they are continuum dominated.

These lines-of-sight offer the possibility of probing large dust column densities in front of extended, parsec-scale pencil beams, contrary to the Milky Way lines-of-sight, where the infrared source is often an embedded object and the resultant spectrum partially contaminated by circumstellar material. It is therefore possible to select extragalactic lines-of-sights free from ice or local carbonaceous absorptions. Moderate extragalactic redshifts also facilitate the ground-based observation of aromatic stretching modes by shifting them into favourable atmospheric windows (Dartois et al. 2007). A bias toward objects dominated by a strongly peaked infrared-emitting source, an AGN, however gives rise to an observational selection effect.

The correlation plot of the silicate optical depth with the $6.85 \mu \mathrm{m}$ feature, when detected, is presented in Fig. 3. We note that there is no straightforward correlation, especially if one considers that the sources where there is little or no hydrocarbon absorption but where strong silicate absorption is observed, are not included in the plot (e.g. IRAS02530+0211). The a-C:H modes are never observed in absorption without the presence of silicates along the line-of-sight. However, the fact that silicates are observed does not mean that the carbonaceous matter can also be observed, the detectivity of the carbonaceous features with respect to the silicates being unfavourable for the former. The correlation plot shows that the carbonaceous grain fraction does not seem to be directly related to the silicate abundance, and therefore might be a separate grain population, as suggested by the polarimetric results. Another possibility is that Fig. 3 is the result of the $\mathrm{H} / \mathrm{C}$ ratio in a-C:H for the different lines-of-sight: if the $\mathrm{H}$ content is reduced, a-C:H tends to a-C, containing fewer $\mathrm{C}-\mathrm{H}$ bonds, and the 6.85 micron feature becomes progressively weaker. In the Milky Way, there are also variations in the a-C:H to extinction ratio, the Galactic centre being about twice as rich in a-C:H as other ISM lines-of-sight, with respect to the visible extinction, as shown by Sandford et al. (1995).

The statistical interest of this plot resides in the confinement of the data to the lower right part of the figure, thus showing that there is a maximum hydrocarbon to silicate ratio, shown as the dotted line with the slope $\tau(6.85) \leq 0.3 \tau$ (Silicate). Using the integrated absorbances of the modes considered here, we can deduce an a-C:H carbon to silicon ratio using:

$\frac{[\mathrm{C}]_{(\mathrm{a}-\mathrm{C}: \mathrm{H})}}{[\mathrm{Si}]_{(\text {Silicates })}} \approx \frac{\tau(6.85) \Delta v(6.85)}{A(6.85)} \times \frac{A(\text { Silicates })}{\tau(\text { Silicates }) \Delta v(\text { Silicates })}$.

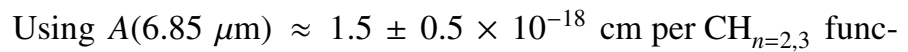
tional group, $\Delta v(6.85) \approx 40 \mathrm{~cm}^{-1}, A($ Silicates $) \approx 1.6 \times$ $10^{-16} \mathrm{~cm} /($ Si atom $), \Delta v($ Silicates $) \approx 300 \mathrm{~cm}^{-1}$, and the above upper limit on the $\tau(6.85) / \tau$ (Silicates) ratio, we obtain:

$\frac{[\mathrm{C}]_{(\mathrm{a}-\mathrm{C}: \mathrm{H})}}{[\mathrm{Si}]_{(\text {Silicates })}}<4.3$.

If we consider that the majority of the cosmically available Silicon is locked into the silicates, this number can be 
E. Dartois and G. M. Muñoz-Caro: Carbonaceous dust grains in luminous infrared galaxies

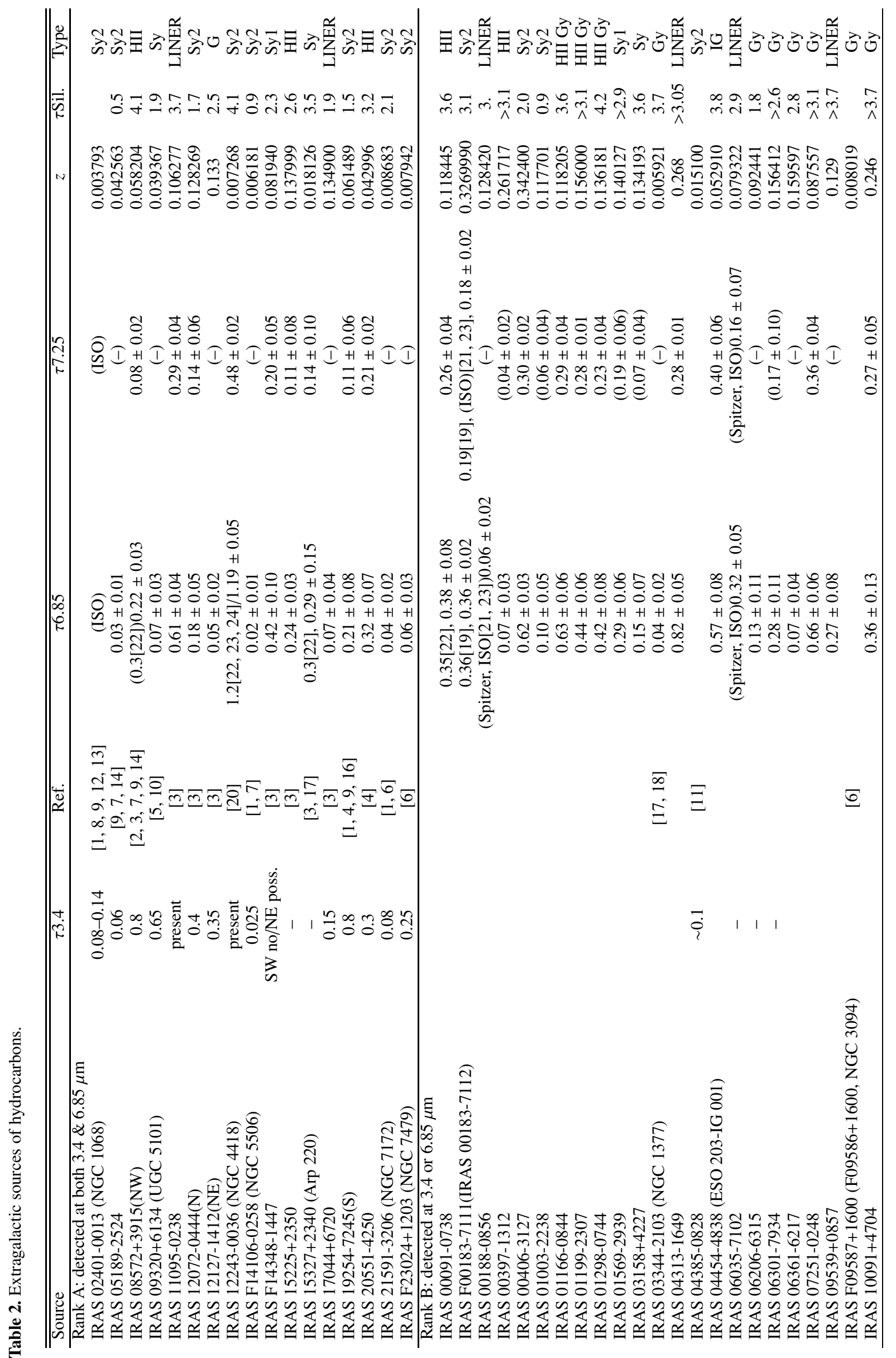




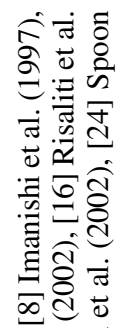

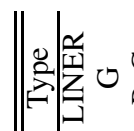
帰

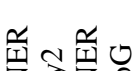

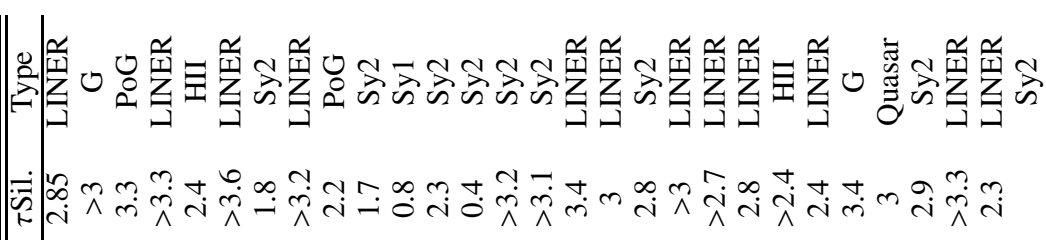

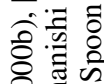

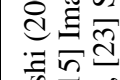
责高通

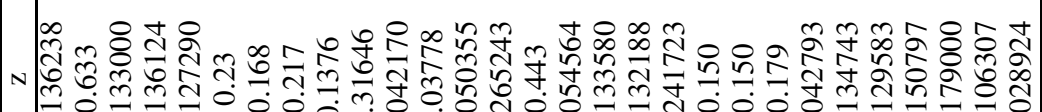

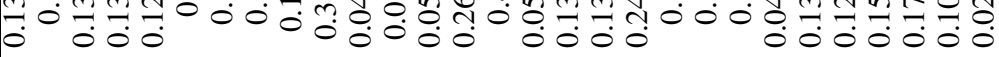

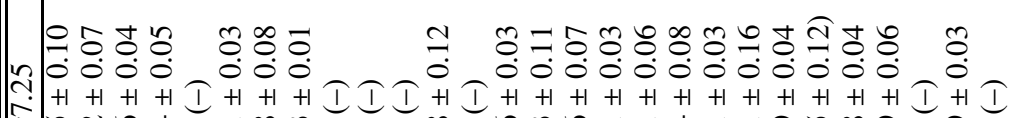

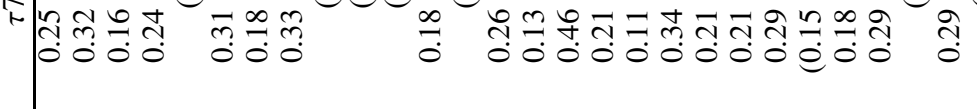

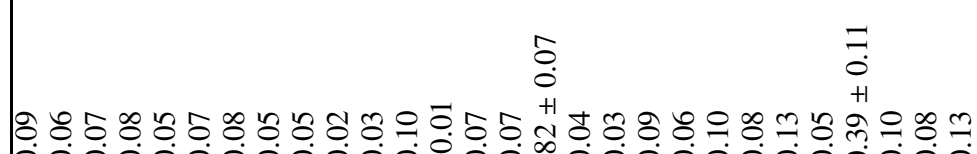

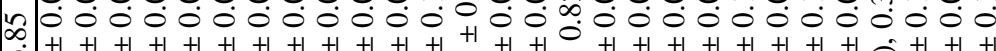

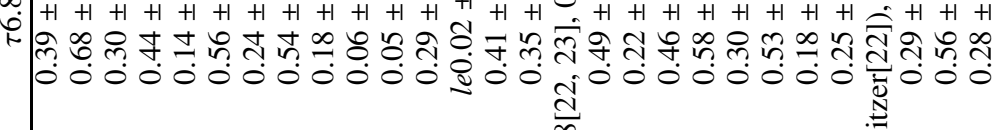
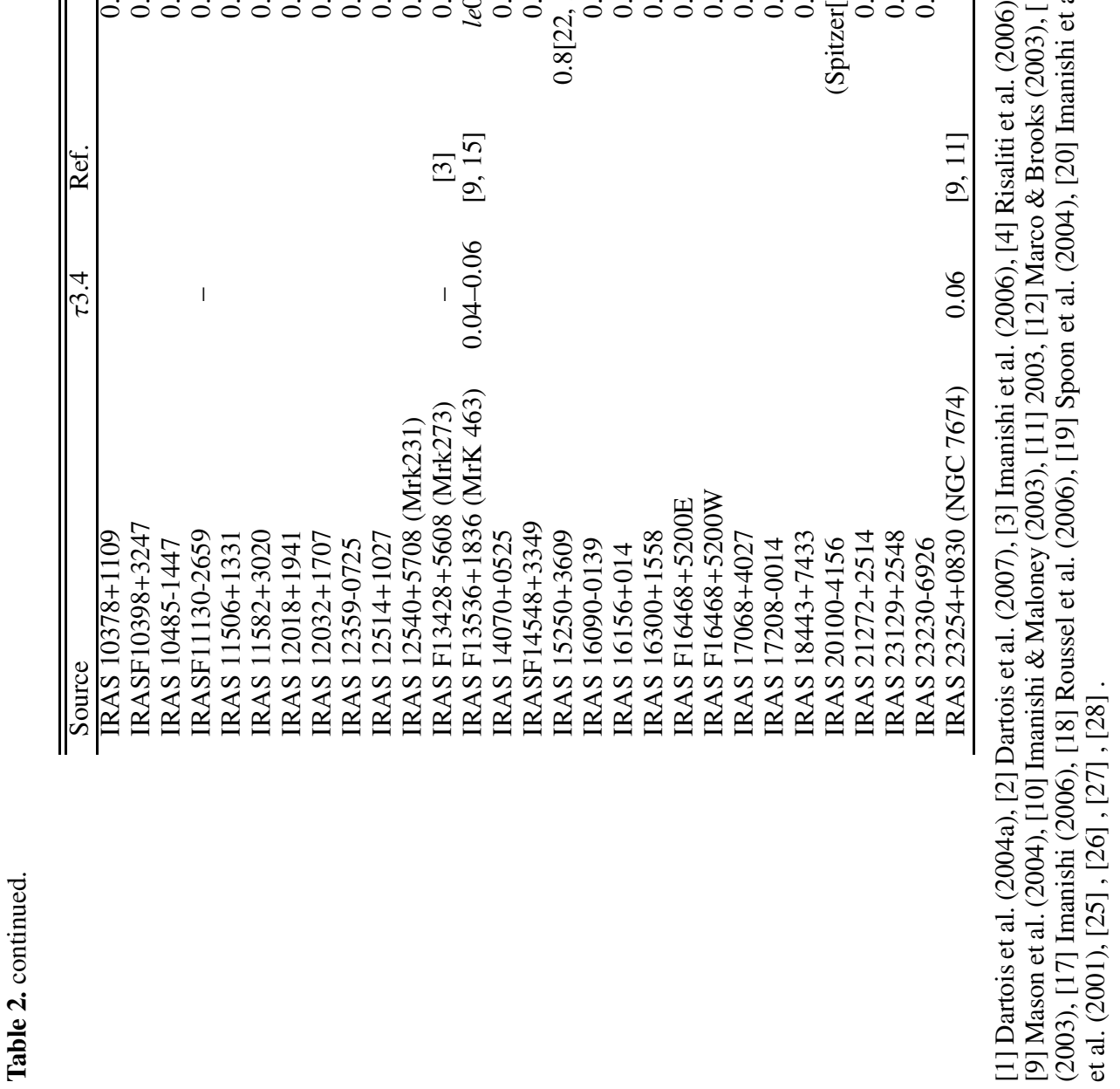


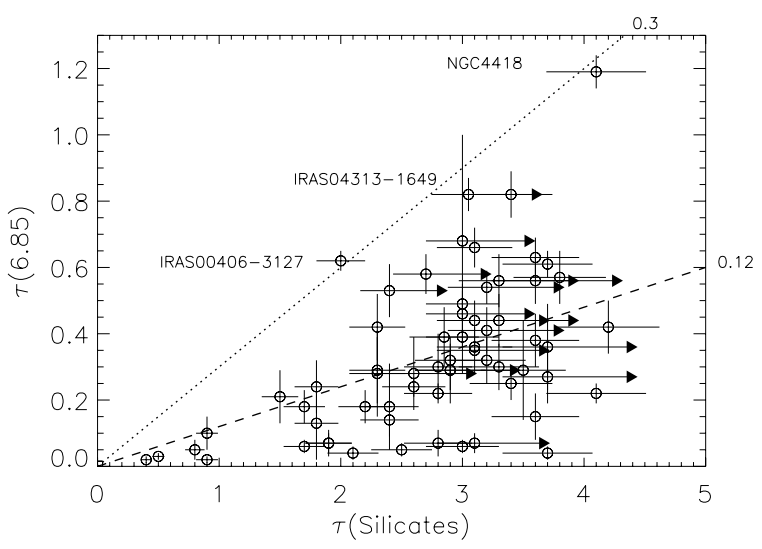

Fig. 3. Correlation of the $6.85 \mu \mathrm{m}$ carbonaceous absorption versus the $9.7 \mu \mathrm{m}$ silicate absorption, obtained from the observed Spitzer spectra presented in Fig. 1. Two linear correlation lines are displayed. The dotted line sets the maximum contribution of a-C:H with respect to silicates (slope 0.3) and the dashed line is the mean expected contribution (slope 0.12) when a-C:H is detected. See text for details.

compared to the cosmic abundance value from various authors: $\frac{[\mathrm{C}]}{[\mathrm{Si}]} \approx 9-11.5$ (Snow \& Witt 1996; Holweger 2001; Sofia 2004). This ratio gives us an estimate of the available carbon locked into the $\mathrm{CH}_{n=2,3}$ functional group of hydrogenated amorphous carbon. We deduce an upper limit to $\frac{[\mathrm{C}]]_{(\mathrm{a}-\mathrm{C}: \mathrm{H})}}{[\mathrm{C}]_{\text {available }}}$ of about $40 \%$, and that the typically observed values span $15 \pm 7 \%$ of the cosmic carbon, in agreement with the Milky Way value (Sect. 3.1, Duley et al. 1998; Sandford et al. 1991). Note that a larger fraction of carbon must be locked up in these grains because more carbon is needed to form the backbone but which does not contribute to the $\mathrm{CH}_{n=2,3}$ absorption bands.

\subsection{The a-C:H galaxy sample}

The observed galaxies represent a subset of the infrared emitting galaxies. They do not display a trend in PAH equivalent width, apart from being globally low intensity PAH emitters, and thus are not starburst dominated. However, the apparent absence of hydrogenated amorphous carbon in other galaxies is almost certainly a selection effect. Strong PAH emission is present in starburst galaxies. PAHs are efficient at re-processing stellar light at moderate visual extinction and will conceal the low contrast absorption arising from the $\mathrm{CH}$ bending modes. The position of the galaxies in our sample, in the Spitzer sample diagram proposed by e.g. Spoon et al. (2007), is not well defined but is skewed toward the upper left side, which also represent the location of sources with the highest column densities.

Very few mid-infrared images with sufficient angular resolution and which could help us to understand the dust configuration have been gathered for the sources with the strongest $6.85 \mu \mathrm{m}$ and $7.25 \mu \mathrm{m}$ absorption features. For NGC 4418, two dark lanes have been observed, extending radially southwest from the nucleus, plus a diffuse one in the northern direction Evans et al. (2003). In the mid-infrared, the spatial extent of the emission is confined to $30-80 \mathrm{pc}$. For IRAS $11095-0238$, the HST I-band image shows a single main body with two clearly resolved cores, and a long, complex tidal tail. It is not obvious whether the two brightest (and also closest) nuclear condensations are in fact multiple nuclei or simply a single core bifurcated by a dense dust lane (Bushouse et al. 2002).
Probably, even with moderate a-C:H absorption, the best studied object in the mid-infrared is the Seyfert 2 galaxy NGC 1068. The observations show that the $3.4 \mu \mathrm{m}$ absorption feature should originate from within a few pc from the centre (Pier \& Krolik 1992). Recent $L$ and $N$ band observations by Tomono et al. (2006) demonstrate that the a-C:H(3.4 $\mu \mathrm{m}) /$ silicate $(9.7 \mu \mathrm{m})$ ratio displays significant spatial variations in the NGC 1068 central region, showing that organic and inorganic matter do not necessarily coexist in the same grains and might be two solid phases evolving separately. Such near- to mid-infrared observations reveal a complex dust cloud distribution around the nucleus, clearly not as the ideal dust torus of the unified model (Antonucci 1993).

\section{Conclusions}

Spitzer IRS extragalactic spectra reveal that the presence of hydrogenated amorphous carbon $(\mathrm{a}-\mathrm{C}: \mathrm{H})$ is common in the ISM of embedded/buried (U)LIRGs, as shown in the large spectroscopic sample of obscured AGNs in this study.

The high column densities of carbon locked into the a-C:H dust observed $\left(>10^{18} \mathrm{~cm}^{-2}\right)$ imply a notable fraction of available cosmic carbon is in this solid phase $(\approx 15 \%)$, in agreement with the Milky Way galactic center lines-of-sight estimates. An even higher fraction of carbon is needed to provide the necessary carbonaceous backbone to the a-C:H structure.

In many extragalactic lines-of-sight the detection of the 6.85 and $7.25 \mu \mathrm{m}$ is hampered by the low contrast inherent in a-C:H absorption, which is mainly detectable through relatively weak infrared active $\mathrm{CH}$ vibrations. The impact of this low absorptivity coupled to the various contributions from other phases/species in the galactic lines-of-sight makes their presence difficult to quantify.

The percentage of interacting galaxies forming and fueling central AGNs with ISM dust might increase at larger redshifts than those of this study. This should favour the detection of the hydrocarbons dust features discussed in this article. It is worth searching for the a-C:H signature in higher $\mathrm{z}$ galaxies, as this ISM carbonaceous dust component is equally as important as the PAH emission carrier.

Acknowledgements. The authors are grateful to Enrik Kruegel for the care with which he reviewed the original manuscript and for constructive criticisms. We also wish to acknowledge Anthony Jones for the numerous suggestions that helped in clarifying the manuscript.

\section{References}

Antonucci, R. 1993, ARA\&A, 31, 473

Armus, L., Charmandaris, V., Bernard-Salas, J., et al. 2007, ApJ, 656, 148 Bridger, A., Wright, G. S., \& Geballe, T. R. 1994, Astronomy with Arrays, The Next Generation, ASSL, 190, 537

Bushouse, H. A., Borne, K. D., Colina, L., et al. 2002, ApJS, 138, 1

Caputi, K. I., et al. 2007, [arXiv: astro-ph/0701283]

Chiar, J. E., Adamson, A. J., Whittet, D. C. B., et al. 2006, ApJ, 651, 268

Dartois, E., Marco, O., Muñoz-Caro, G. M., et al. 2004a, A\&A, 423, 549

Dartois, E., Muñoz Caro, G. M., Deboffle, D., \& d'Hendecourt, L. 2004b, A\&A, 423, L33

Dartois, E., Muñoz Caro, G. M., Deboffle, D., Montagnac, G., \& D’Hendecourt, L. 2005, A\&A, 432, 895

Dartois, E., Geballe, T. R., Pino, T., et al. 2007, A\&A, 463, 635

Dole, H., Lagache, G., Puget, J.-L., et al. 2006, A\&A, 451, 417

Draine, B. T. 2003, ARA\&A, 41, 241

Duley, W. W., Scott, A. D., Seahra, S., \& Dadswell, G. 1998, ApJ, 503, L183

Evans, I. N., Ford, H. C., Kinney, A. L., et al. 2003, ApJ, 369, L27

Genzel, R., Lutz, D., Sturm, E., et al. 1998, ApJ, 498, 579

Greenberg, J. M., Li, A., Mendoza-Gomez, C. X., et al. 1995, ApJ, 455, L177 
Gratadour, D., Clénet, Y., Rouan, D., Lai, O., \& Forveille, T. 2003, A\&A, 411, 335

Higdon, S. J. U., Devost, D., Higdon, J. L., et al. 2004, PASP, 116, 975

Holweger, H. 2001, Joint SOHO/ACE workshop Solar and Galactic Composition, 598, 23

Houck, J. R., Roellig, T. L., van Cleve, J., et al. 2004, ApJS, 154, 18

Imanishi, M. 2000a, MNRAS, 319, 331

Imanishi, M. 2000b, MNRAS, 313, 165

Imanishi, M. 2002, ApJ, 569, 44

Imanishi, M. 2003, ApJ, 599, 918

Imanishi, M. 2006, AJ, 131, 2406

Imanishi, M., \& Dudley, C. C. 2000, ApJ, 545, 701

Imanishi, M., \& Maloney, P. R. 2003, ApJ, 588, 165

Imanishi, M., Terada, H., Sugiyama, K., et al. 1997, PASJ, 49, 69

Imanishi, M., Dudley, C. C., \& Maloney, P. R. 2001, ApJ, 558, L93

Imanishi, M., Nakanishi, K., Kuno, N., \& Kohno, K. 2004, AJ, 128, 2037

Imanishi, M., Dudley, C. C., \& Maloney, P. R. 2006, ApJ, 637, 114

Imanishi, M., Dudley, C. C., Maiolino, R., et al. 2007, ApJS, 171, 72

Lutz, D., Spoon, H. W. W., Rigopoulou, D., Moorwood, A. F. M., \& Genzel, R. 1998, ApJ, 505, L103

Marco, O., \& Brooks, K. J. 2003, A\&A, 398, 101

Mason, R. E., Wright, G., Pendleton, Y., \& Adamson, A. 2004, ApJ, 613, 770

Mason, R. E., Wright, G. S., Adamson, A., \& Pendleton, Y. 2007, ApJ, 656, 798

Pendleton, Y. J., Sandford, S. A., Allamandola, L. J., Tielens, A. G. G. M., \& Sellgren, K. 1994, ApJ, 437, 683
Pier, E. A., \& Krolik, J. H. 1992, ApJ, 401, 99

Rigopoulou, D., Spoon, H. W. W., Genzel, R., et al. 1999, AJ, 118, 2625

Risaliti, G., Maiolino, R., Marconi, A., et al. 2003, ApJ, 595, L17

Risaliti, G., Maiolino, R., Marconi, A., et al. 2006, MNRAS, 365, 303

Roussel, H., et al. 2006, ApJ, 646, 841

Ryter, C. E. 1996, Ap\&SS, 236, 285

Sajina, A., Yan, L., Armus, L., et al. 2007, ApJ, 664, 713

Sandford, S. A., Pendleton, Y. J., \& Allamandola, L. J. 1995, ApJ, 440, 697

Sandford, S. A., Allamandola, L. J., Tielens, A. G. G. M., et al. 1991, ApJ, 371, 607

Snow, T. P., \& Witt, A. N. 1996, ApJ, 468, L65

Sofia, U. J. 2004, Astrophysics of Dust, 309, 393

Spoon, H. W. W., Keane, J. V., Tielens, A. G. G. M., Lutz, D., \& Moorwood, A. F. M. 2001, A\&A, 365, L353

Spoon, H. W. W., Keane, J. V., Tielens, A. G. G. M., et al. 2002, A\&A, 385, 1022 Spoon, H. W. W., Armus, L., Cami, J., et al. 2004, ApJS, 154, 184

Spoon, H. W. W., Keane, J. V., Cami, J., et al. 2005, IAU Symp., 231, 281

Spoon, H. W. W., Tielens, A. G. G. M., Armus, L., et al. 2006, ApJ, 638, 759

Spoon, H. W. W., Marshall, J. A., Houck, J. R., et al. 2007, ApJ, 654, L49

Tomono, D., Terada, H., \& Kobayashi, N. 2006, ApJ, 646, 774

Tran, Q. D., Lutz, D., Genzel, R., et al. 2001, ApJ, 552, 527

Werner, M. W., Roellig, T. L., Low, F. J., et al. 2004, ApJS, 154, 1

Wexler, A. S. 1967, Appl. Spectroscopy Rev., 1, 29 